\title{
Hybrid Interferometric/Dispersive Atomic Spectroscopy of Laser-induced Uranium Plasma
}

\author{
Phyllis K. Morgan \\ The Pennsylvania State University, University Park, PA 16802, USA \\ Jill R. Scott \\ Idaho National Laboratory, Idaho Falls, ID 83415, USA \\ Igor Jovanovic ${ }^{1}$ \\ The Pennsylvania State University, University Park, PA 16802, USA
}

\begin{abstract}
An established optical emission spectroscopy technique, laser-induced breakdown spectroscopy (LIBS), holds promise for detection and rapid analysis of elements relevant for nuclear safeguards, nonproliferation, and nuclear power, including the measurement of isotope ratios. One such important application of LIBS is the measurement of uranium enrichment $\left({ }^{235} \mathrm{U} /{ }^{238} \mathrm{U}\right)$, which requires high spectral resolution (e.g., $25 \mathrm{pm}$ for the 424.4 $\mathrm{nm}$ U II line). High-resolution dispersive spectrometers necessary for such measurements are typically bulky and expensive. We demonstrate the use of an alternative measurement approach, which is based on an inexpensive and compact Fabry-Perot etalon integrated with a low to moderate resolution Czerny-Turner spectrometer, to achieve the resolution needed for isotope selectivity of LIBS of uranium in ambient air. Spectral line widths of $\sim 10 \mathrm{pm}$ have been measured at a center wavelength $424.437 \mathrm{~nm}$, clearly discriminating the natural from the highly enriched uranium.
\end{abstract}

Keywords: Laser-induced breakdown spectroscopy, Uranium, Isotopes, Fabry-Perot

${ }^{1}$ Corresponding author: ijovanovic@psu.edu

\section{Introduction}

Laser-induced breakdown spectroscopy (LIBS) enables nearly instantaneous material composition analysis with high spatial resolution and essentially no sample preparation. Rapid elemental and isotopic characterization of materials relevant to nuclear safeguards is desirable. LIBS is advantageous in such applications because it is unaffected by ionizing radiation and compatible with measurements at a large standoff. Unlike LIBS, techniques such as X-ray fluorescence spectroscopy can be affected by the presence of gamma radiation [1]. Furthermore, LIBS can be used to measure non-radioactive sources. Filamentation LIBS can be used to conduct measurements over long distances, in situations where laser beam focusing onto a surface can be challenging (e.g., in highly radioactive areas or in facilities with limited access) [2]. Because LIBS directly samples the material surface, it requires the analyte to be accessible within the field of view of the laser source. The potential for use of LIBS for analysis of nuclear fuel composition, waste storage, and reactor process monitoring has been explored for its real-time, in-situ capability to verify inventory and characterize nuclear materials, even in harsh 
environments $[3,4,5]$. Experiments have been conducted to study the effects of experimental parameters, including laser pulse duration and shape, in order to improve sensitivity and reproducibility in LIBS of nuclear materials [6,7].

One of the challenges when making isotopic measurements by LIBS is the physical broadening of emission peak widths, which can approach or exceed the isotope shift. In a LIBS plasma, Doppler and Stark effect are the predominant mechanisms of peak broadening and are influenced by the gas environment surrounding the plasma $[8,9]$. Another challenge is of technological nature. Isotope measurements usually require highresolution spectrometers and temporally gated detectors to resolve the isotope shift. In previous LIBS studies, the isotope shift between ${ }^{235} \mathrm{U}$ and ${ }^{238} \mathrm{U}$ of $25 \mathrm{pm}$ at the 424.437 $\mathrm{nm}$ atomic emission line has been measured [15-18]. In the study of uranium by Pietsch et al., a $1 \mathrm{~m}$ focal length spectrometer was used with a single channel detector and a boxcar integrator, operating as a monochromator scanning in $1 \mathrm{pm}$ increments. The measurement was made under 2.67 Pa pressure [10]. Smith et al. measured the $0.05 \mathrm{~nm}$ isotope shift between ${ }^{239} \mathrm{Pu}$ and ${ }^{240} \mathrm{Pu}$ at the $594.5 \mathrm{~nm}$ emission line, under a $13.3 \mathrm{kPa}$ helium atmosphere, using a $2 \mathrm{~m}$ focal length double-pass spectrometer with an intensified charge-coupled device (ICCD) [11]. LIBS has also been used to study isotopes of uranium and hydrogen, in air at atmospheric pressure, using a $0.55 \mathrm{~m}$ focal length Czerny-Turner spectrometer coupled to an ICCD [12]. The individual peaks of the uranium isotopes were not resolved. Instead, partial least squares analysis was successfully applied to predict a range of uranium samples with different enrichments. Cremers et al. observed the uranium isotope shift with high-resolution spectrometers [13]. This study was based on a LIBS system with compact, portable laser delivery and light collecting optics coupled to one of two relatively expensive laboratory echelle spectrometers. This LIBS system was able to distinguish the isotopes of uranium, hydrogen, and lithium with careful experimental timing.

Isotope analysis of uranium is also possible by alternative laser-ablation sampling analysis such as laser-induced fluorescence $[14,15,16]$ and laser absorption spectrometry $[17,18]$. For example, Liu et al. used laser absorption to measure the ${ }^{235} \mathrm{U} /{ }^{238} \mathrm{U}$ isotope ratio at $682.0768 \mathrm{~nm}(-12.8 \mathrm{pm}$ isotope shift). A negative value of the isotope shift indicates that the ${ }^{235} \mathrm{U}$ wavelength is greater than that of ${ }^{238} \mathrm{U}$, and vice versa [15]. Smith et al. used laser-induced fluorescence to distinguish between the uranium isotopes at $683.2719 \mathrm{~nm}$ (16.8 pm shift) [17]. A disadvantage of these approaches is that multiple lasers and detectors are required for probing and analyzing the plasma plume. With a single laser for sampling and analysis and a single detector, LIBS is comparatively simpler.

Additionally, laser ablation molecular isotopic spectrometry (LAMIS) was developed to make isotope measurements [19]. Molecular emission spectra can exhibit greater isotope shifts than atomic emission spectra, relaxing the requirements on spectral resolution. For example, the atomic isotope shift for B is $0.002 \mathrm{~nm}$ and the observed isotope shift of BO is $0.73 \mathrm{~nm}$ [20]. It has also been shown that the use of a non-gated detector can be sufficient for accurate isotope characterization in LAMIS [21,22]. While the resolution requirement may not be as stringent, the signal-to-noise for molecular peaks is often poor and molecular spectra typically require mathematical processing that involve 
chemometrics. Furthermore, molecular measurements are limited to those elements that combine to form molecular species. Because molecular rotations and vibrations are mass dependent and isotope shifts scale with the reduced mass of the molecular species, heavier atoms exhibit smaller shifts [21]. A molecular isotope shift in LIBS spectra for uranium has not yet been reported.

Effenberger and Scott proposed an alternative method for performing high-resolution LIBS measurements by integrating a low-cost Fabry-Perot (FP) etalon with a $0.5 \mathrm{~m}$ focal length Czerny-Turner spectrometer [23]. Instrument performance was demonstrated using the hyperfine doublet of the $313.2 \mathrm{~nm}$ mercury emission line, which exhibits a splitting of $29 \mathrm{pm}$, similar to an isotope shift of uranium. Recently, a method was developed to reconstruct the high-resolution spectrum from a similar hybrid interferometric/dispersive LIBS instrument and showed improvement in spectral resolution and accurate determination of peak ratios compared to conventional pixel-towavelength schemes [24].

In this study, we demonstrate for the first time the capability of a hybrid interferometric/dispersive instrument to achieve isotope selectivity in LIBS of uranium. The results of this study could help advance LIBS applications in the field where highresolution is needed. Compact and cost-effective technologies capable of isotope measurements could benefit nuclear safeguards verification and nonproliferation as well as other high-resolution spectroscopic applications.

\section{Experimental}

\subsection{Samples}

The samples used were a natural uranium foil $\left(0.7 \%\right.$ of $\left.{ }^{235} \mathrm{U}\right)$ and a uranium foil highly enriched in ${ }^{235} \mathrm{U}$ to $93 \%$. Both samples were provided by the Penn State University's Breazeale Nuclear Reactor.

\subsection{Experimental setup}

A diagram of the experimental setup is shown in Fig. 1. The samples were placed in a sealed experimental chamber under atmospheric pressure and mounted to a sample holder at normal incidence to the laser beam. The sealed chamber was used strictly for containment of the ablated uranium. The laser pulse width was $10 \mathrm{~ns}$ at a wavelength of $1064 \mathrm{~nm}$ generated by a Nd:YAG laser (Spectra Physics). The laser pulses were focused onto the sample through an optical window using a $300 \mathrm{~mm}$ focal length lens. The emission from the plasma was collected and collimated by a $5 \mathrm{~cm}$ focal length lens mounted off-axis to the laser beam path. A second lens focused the collimated light into a $400 \mu \mathrm{m}$ core diameter optical fiber (Ocean Optics), which transported the light to the broadband etalon (custom built by SLS Optics). The FP etalon was aligned to the entrance slit (500 $\mu \mathrm{m}$ width) of the spectrometer. A $25 \mathrm{~mm}$ diameter lens with a focal length of $150 \mathrm{~mm}$ was used to focus the light transmitted by the etalon onto the entrance slit of the spectrometer (Horiba Jobin Yvon), which had a $550 \mathrm{~mm}$ focal length and a 
$1800 \mathrm{~mm}^{-1}$ grating coupled to a $1024 \mathrm{x} 1024$ pixel array iStar ICCD (Andor) with $13 \mu \mathrm{m}$ pixel size. Instrument alignment and spectral calibration was performed using an $\mathrm{Hg}(\mathrm{Ar})$ pen lamp (Oriel) acquired with $1 \mathrm{~s}$ ICCD exposures for each snapshot (10 snapshots per image), at $24.4 \%$ of the maximum detector gain.

\subsection{LIBS parameters}

For LIBS measurements, the plasma was generated in ambient atmosphere using $30 \mathrm{~mJ}$ laser pulses. For conventional LIBS without the FP etalon, spectra were measured using the $0.55 \mathrm{~m}$ Czerny-Turner spectrometer with an entrance slit width of $13 \mu \mathrm{m}$, ICCD gate width of $1 \mu \mathrm{s}$ at a delay of $5 \mu \mathrm{s}$, and gain set at $<1 \%$ of the maximum gain. Each spectrum is the average of 5 single-shot spectra. The sample was translated between each spectrum measurement, and cleaning shots were made prior to each measurement to remove oxidized uranium on the sample surface [8]. Because of the lower throughput of the high-resolution hybrid instrument (estimated to be $<0.01 \%$ for the sampled portion of ring pattern compared to the Czerny-Turner spectrometer alone), each spectrum was collected from 600 laser shots ( 1 min exposure at a $10 \mathrm{~Hz}$ pulse rate). Because of the high repetition rate, the sample was not translated between each pulse during the 1 min exposure. The gate time was set to $10 \mu \mathrm{s}$ with $5 \mu$ s delay, and ICCD gain was set to $97.6 \%$ of the maximum gain.

\section{Results and discussion}

\subsection{Spectral reconstruction}

The broadband pattern from a FP etalon is composed of superimposed, angularly and spectrally dependent interference fringes that are sampled by the spectrometer entrance slit, separated by the grating, and detected on the pixel array to produce a slice of the fringe pattern (Fig. 2(a)). A spectral reconstruction method was developed to analyze the high-resolution fringes incident on the detector without presuming the distribution of the source spectrum. The mathematical model used for reconstructing the spectrum from a FP fringe pattern has been described and validated previously [24]. The accuracy of the reconstruction depends on proper alignment of the optical fiber, Fabry-Perot etalon and the entrance slit of the spectrometer. An optics laboratory presents a stable, low-drift environment that did not require realignment of the instrument over the time during which the experiment was conducted (a span of several weeks). In an instrument deployed in the field based on this technique, the optical system would need to be carefully engineered for robustness to shock, vibration, and effect of temperature drift. The complexity of engineering needed to achieve stability in field applications is not expected to exceed that routinely achieved in existing optical systems deployed in harsh environments.

\subsection{Calibration}

The hybrid interferometric/dispersive spectrometer was aligned using the $435.84 \mathrm{~nm}$ line of an $\mathrm{Hg}(\mathrm{Ar})$ calibration lamp because of its proximity to the uranium lines of interest. 
The angular $(\theta)$ axis of the $435.84 \mathrm{~nm}$ interference pattern image was calibrated by a linear fit, as shown in Fig. 2(b). In Fig. 2(c), etalon reflectivity was determined to be $R=$ 0.77 by fitting the measured intensity profile of the $\mathrm{Hg} 435.84 \mathrm{~nm}$ line from a lamp to the FP etalon transmission function, corresponding to a resolution of $6 \mathrm{pm}$ at $424.437 \mathrm{~nm}$. The intensity profile was obtained by summing along the horizontal (wavelength) axis of the image. Only half the interference pattern was analyzed due to cylindrical symmetry of the interference rings. The angular dependence of the intensity distribution (beam profile) was taken into account by convolving the fitting function with a Gaussian function. Fig. 2(a) represents an accumulation of 10 images.

\subsection{Uranium analysis}

Performance of a conventional and hybrid spectrometer are compared to understand the tradeoffs between sensitivity and resolution for measuring the ${ }^{235} \mathrm{U} /{ }^{238} \mathrm{U}$ isotope shift. Fig. 3 shows the standard LIBS spectra comparing natural and highly enriched uranium (HEU) in the vicinity of the U II $424.437 \mathrm{~nm}$ isotope shift. The resolution of the $0.55 \mathrm{~m}$ Czerny-Turner spectrometer used in this measurement (70 pm) was apparently insufficient for distinguishing between ${ }^{235} \mathrm{U}$ and ${ }^{238} \mathrm{U}$ lines.

The isotopically resolved measurement of uranium using a hybrid interferometric/dispersive spectrometer is shown in Fig. 4. The profile of the fringes comparing natural and HEU are shown in Fig. 5. Calibration of the angular $(\theta)$ axis was based on the interference pattern produced by the ${ }^{238} \mathrm{U}$ lines. When a portion of the uranium $424.167 \mathrm{~nm}$ and $424.437 \mathrm{~nm}$ ring pattern was integrated, the isotope shift of the lines was distinguishable (Fig. 5). Even without application of further mathematical analysis, the isotope shifts of the $424.167 \mathrm{~nm}$ and 424.437 lines were easily measured to be $-6 \mathrm{pm}$ and $25 \mathrm{pm}$, respectively, which agrees with previously observed values [14]. The $5 \mathrm{pm}$ isotope shift of the U I $424.626 \mathrm{~nm}$ line was not studied due to partial overlap of the $424.626 \mathrm{~nm}$ and $424.412 \mathrm{~nm}$ interference patterns.

To further validate the resolution in the isotope shift measurements, experimental data was interpolated and resampled to improve the sampling rate for reconstruction via the inversion method [24]. A low-pass filtering of the experimental data was also applied prior to reconstruction of the source spectrum to reduce the random spectral noise. The isotopically shifted spectral lines, reconstructed using the inversion method, are shown in Fig. 6. A tolerance of 0.3 was used to minimize error in the reconstruction. In order to generate the fringe profile of the $424.167 \mathrm{~nm}$ and its isotope shift, the 2-D image was summed along the $\mathrm{x}$-axis in such a way that the patterns from the two lines were isolated (spectrum not shown). The ranges of $\theta$ and $\lambda$ used to define the instrument function $\mathbf{T}_{\theta, \lambda}$ for reconstructing the $424.167 \mathrm{~nm}$ line in Fig. 6(a) were $16 \mathrm{mrad}<\theta<19 \mathrm{mrad}$ and $424.137 \mathrm{~nm}<\lambda<424.197 \mathrm{~nm}$. For the same figure, $15 \mathrm{mrad}<\theta<18 \mathrm{mrad}$ and $424.143 \mathrm{~nm}<\lambda<424.203 \mathrm{~nm}$ were used to define $\mathbf{T}_{\theta, \lambda}$ for the $424.173 \mathrm{~nm}$ line. The $424.437 \mathrm{~nm}$ line and its isotope shift were reconstructed from a fringe profile obtained by summing along a portion of rings that included both the $424.167 \mathrm{~nm}$ and $424.437 \mathrm{~nm}$ interference rings. This could not be avoided due to the overlap of the ring patterns (Fig. 4). For reconstructing the $424.437 \mathrm{~nm}$ line, the following constraints were used: $22<\theta$ 
$<23.5 \mathrm{mrad}$ and $424.362 \mathrm{~nm}<\lambda<424.462 \mathrm{~nm}$. For the $424.412 \mathrm{~nm}$ line, $19 \mathrm{mrad}<\theta$ $<20.5 \mathrm{mrad}$ and $424.387 \mathrm{~nm}<\lambda<424.487 \mathrm{~nm}$ were used to define $\mathbf{T}_{\theta, \lambda}$. Each line was reconstructed separately to avoid interference from adjacent spectral lines. Spectral line widths of $\sim 10 \mathrm{pm}$ were measured at $424.437 \mathrm{~nm}$ and the $25 \mathrm{pm}$ isotope shift was well resolved, while the $-6 \mathrm{pm}$ shift of the $424.167 \mathrm{~nm}$ line was partially discernible. The widths of the ${ }^{235} \mathrm{U}$ lines are broader than ${ }^{238} \mathrm{U}$ lines because, unlike ${ }^{235} \mathrm{U},{ }^{238} \mathrm{U}$ is an eveneven isotope with zero nuclear angular momentum, which does not exhibit hyperfine structure that would broaden or split its spectral lines [25].

In previous work by Cremers et al., the isotope shift of uranium was also measured in ambient air [14]. The isotope shift of the $424.437 \mathrm{~nm}$ line was observed with spectrometer resolution $<10 \mathrm{pm}$. In uranium measurements made with a DEMON spectrometer ( $6.8 \mathrm{pm}$ resolution) coupled to an ICCD, the $25 \mathrm{pm}$ isotope shift at $424.437 \mathrm{~nm}$ was well resolved and the $-6 \mathrm{pm}$ isotope shift at $424.167 \mathrm{~nm}$ was partially resolved. Each spectrum measured was composed of 100 laser shots. In that work, an EMU-65 spectrometer ( $10 \mathrm{pm}$ resolution) distinguished between the lines of the $25 \mathrm{pm}$ isotope shift of the $424.4 \mathrm{~nm} \mathrm{U}$ II line with a non-gated detector, although the spectra were pixilated due to the lower resolution. Each EMU-65 spectrum was an average of 60 plasmas from the sample. Because each frame taken lasted approximately $1 \mathrm{~s}$, the collection time was 1 min per spectrum. In comparison, the resolution achieved by the hybrid interferometric/dispersive spectrometer is similar to the resolution of the DEMON spectrometer. However, spectrum taken by the hybrid instrument required relatively longer collection times, similar to collection times of the EMU-65 for uranium ( 1 min). The current laboratory setup of the hybrid instrument is approximately the same size of DEMON and larger than the EMU-65. While the resolution of the instrument results from the combination of characteristics of all components of the light collection and detection system, the vast majority of the resolving power results from the use of the FP etalon [24]. Thus, replacing the $0.55 \mathrm{~mm}$ focal length spectrometer of the hybrid device with a more compact dispersive device, such as a miniature spectrometer, could substantially reduce the overall footprint of the instrument without sacrificing performance in this application.

\section{Conclusion}

LIBS measurements of uranium isotopes using a hybrid interferometric/dispersive spectrometer were demonstrated and high-resolution wavelength spectra of uranium spectra were retrieved. Summation of entire patterns from different lines of uranium produced by the FP etalon may be able to increase the signal-to-background, but was not implemented here due to the partial overlap of the U I 424.626 line and U II $424.412 \mathrm{~nm}$ lines. With a resolution of $\sim 6 \mathrm{pm}$, the instrument resolved the $25 \mathrm{pm}$ isotope shift at $424.437 \mathrm{~nm}$ for uranium in ambient air. However, the $424.167 \mathrm{~nm}$ isotope shift, limited by spectral broadening, was only partially resolved. Measurements at reduced pressures or in other ambient gases could be used to reduce the spectral broadening, but were beyond the scope of this study. Obtaining uranium samples with a broader intermediate range of enrichments was challenging and consequently not pursued in this work. As a result, characterization of isotope ratio accuracy of the instrument is recommended for 
future study. Integrating a FP etalon with a dispersive spectrometer has proved to be an inexpensive and effective alternative to $2 \mathrm{~m}$ focal length Czerny-Turner or echelle spectrometers for detection of uranium isotopes with LIBS. Although these measurements were made in a laboratory setting, the results of this work could eventually lead to the development of portable, low-cost, and high-resolution LIBS instruments with isotopic-measurement capability.

\section{Acknowledgements}

The authors would like to thank Eric Boeldt, Jeff Leavey, and the staff at the Penn State Radiation Science and Engineering Center for their help with obtaining uranium samples. Research was performed under appointment to the Nuclear Nonproliferation International Safeguards Graduate Fellowship Program sponsored by the National Nuclear Security Administration's Next Generation Safeguards Initiative (NGSI). Material is based upon work supported by the U.S. Department of Homeland Security under Grant Award Number, 2012-DN-130-NF0001-02. The views and conclusions contained in this document are those of the authors and should not be interpreted as necessarily representing the official policies, either expressed or implied, of the U.S. Department of Homeland Security. Research was also sponsored by the U.S. Department of Energy under DOE Idaho Operations Office Contract DE-AC07-05ID14517. This work was funded in-part by the Consortium for Verification Technology under the Department of Energy National Nuclear Security Administration, award number DE-NA0002534. 


\section{References}

[1] A.I. Whitehouse, J. Joung, I.M. Botheroyd, S. Lawson, C. Evans, J. Wright, Remote material analysis of nuclear power station steam generator tubes by laser-induced breakdown spectroscopy, Spectrochim. Acta, Part B 56 (2001) 821-830. doi:10.1016/S0584-8547(01)00232-4

[2] K. Stelmaszczyk, P. Rohwetter, G. Mejean, J. Yu, E. Salmon, J. Kasparian, R. Ackermann, J. Wolf, L. $\mathrm{W} \square$ ost, Long-distance remote laser-induced breakdown spectroscopy using filamentation in air, Appl.

Phys. Lett. 85 (18) (2004) 3977-3979. doi:10.1063/1.1812843

[3] C. Hanson, S. Phongikaroon, J.R. Scott, Temperature effect on laser- induced breakdown spectroscopy spectra of molten and solid salts, Spectrochim. Acta, Part B 97 (2014) 79-85.

doi:10.1016/j.sab.2014.04.012

[4] M. Tampo, M. Miyabe, K. Akaoka, M. Oba, H. Ohba, Y. Maruyama, I. Wakaida, Enhancement of intensity in microwave-assisted laser induced-breakdown spectroscopy for remote analysis of nuclear fuel recycling J. Anal. At. Spectrom. 29 (2014) 886-892. doi:10.1039/ C3JA50259G

[5] X.K. Shen, Y.F. Lu, Detection of uranium in solids by using laser-induced breakdown spectroscopy combined with laser-induced fluorescence, Appl. Opt. 47 (2008) 1810-1815. doi:10.1364/AO.47.001810

[6] L.A. Emmert, R.C. Chinni, D.A. Cremers, C.R. Jones, W. Rudolph, Comparative study of femtosecond and nanosecond laser-induced breakdown spectroscopy of depleted uranium, Appl. Opt. 50 (3) (2011) 313317. doi:10.1364/AO.50.000313

[7] P. Ko, K.C. Hartig, J.P. McNutt, R.B.D. Schur, T.W. Jacomb-Hood, I. Jovanovic, Adaptive femtosecond laser-induced breakdown spectroscopy of uranium, Rev. Sci. Instrum. 84 (2013) 013104. doi:10. 1063/1.4779042

[8] A.J. Effenberger Jr., J.R. Scott, Effect of atmospheric conditions on LIBS spectra, Sensors 10 (2010) 4907-4925. doi:10.3390/s100504907

[9] J.R. Scott, A.J. Effenberger Jr., J.J. Hatch, Laser-induced Breakdown Spectroscopy -Theory and Applications, Springer-Verlag, New York, 2014 (Chapter4).

[10] W. Pietsch, A. Petit, A. Briand, Isotope ratio determination of uranium by optical emission spectroscopy on a laser-produced plasma - basic investigations and analytical results, Spectrochim. Acta, Part B 53 (1998) 751-761. doi:10.1016/S0584-8547(97)00123-7

[11] C.A. Smith, M.A. Martinez, D.K. Veirs, D.A. Cremers, Pu-239/Pu-240 isotope ratios determined using high resolution emission spectroscopy in a laser-induced plasma, Spectrochim. Acta, Part B 57 (2002) 929937. doi:10.1016/S0584-8547(02)00023-X

[12] F.R. Doucet, G. Lithgow, R. Kosierb, P. Bouchard, M. Sabsabi, Determination of isotope ratios using laser-induced breakdown spectroscopy in ambient air at atmospheric pressure for nuclear forensics, J. Anal. At. Spectrom. 26 (2011) 536-541. doi:10.1039/C0JA00199F

[13] D.A. Cremers, A. Beddingfield, R. Smithwick, R.C. Chinni, C.R. Jones, B. Beardsley, L. Karch, Monitoring uranium, hydrogen, and lithium and their isotopes using a compact laser-induced breakdown spectroscopy (LIBS) probe and high-resolution spectrometer, Appl. Spectrosc. 66 (3) (2012) 250-261. doi:10.1039/C0JA00199F.

[14] L. Liu, S. Li, X. Huang, Y. Lu, K. Chen, R. Pik, L. Jiang, J.F. Silvaine, Y.F. Lu, Detection of tracelevel uranium and samarium in glasses by combined laser-induced breakdown spectroscopy and plasmainduced fluorescence spectroscopy, J. Anal. At. Spectrom. 30 (2015) 1128-1132. doi: 10.1039/c5ja00020c 
[15] M. Oba, K. Akaoka, M. Miyabe, I. Wakaida, Isotope shift and hyperfine structure of the highly excited atom uranium, The Eur. Phys. J. D, 21 (2002) 255-260. doi:/10.1140/epjd/e2002-00215-y

[16] B.W. Smith, A. Quentmeier, M. Bolshov, K. Niemax, Measurement of uranium isotope ratios in solid samples using laser ablation and diode laser-excited atomic fluorescence spectrometery, Spectrochim. Acta, Part B 54 (1999) 943-958. doi:10.1016/S0584-8547(99)00022-1

[17] H. Liu, A. Quentmeier, K. Niemax, Diode laser absorption measurement of uranium isotope ratios in solid samples using laser ablation, Spectrochim. Acta, Part B 57 (2002) 1611-1623. doi:10.1016/S0584$8547(02) 00105-2$

[18] A. Quentmeier, M. Bolshov, K. Niemax, Measurement of uranium isotope ratios in solid samples using laser ablation and diode laser-atomic absorption spectrometry, Spectrochim. Acta, Part B 56 (2001) 45-55. doi:10.1016/S0584-8547(00)00289-5

[19] H. Niki, Yasuda, I. Kitazima, Measurement technique of boron isotopic ratio by laser-induced breakdown spectroscopy, J. Nucl. Sci. and Technol. 35 (1998) 34-39.

doi:10.1080/18811248.1998.9733817.

[20] R.E. Russo, A.A. Bolshakov, X. Mao, C.P. McKay, D.L. Perry, O. Sorkhabi, Laser ablation molecular isotopic spectrometry, Spectrochim. Acta, Part B 66 (2011) 99-104. doi:10.1016/j.sab.2011.01.007.

[21] B. Yee, K.C. Hartig, P. Ko, J. P. McNutt, I. Jovanovic, Measurement of boron isotopic ratio with nongated molecular spectroscopy of femtosecond laser-produced plasma, Spectrochim. Acta, Part B 78-79 (2013) 72-76. doi:10.1016/j.sab.2012.12.003

[22] P. Ko, I. Jovanovic, Boron isotopic measurements from spectrally filtered non-gated molecular spectra induced by laser ablation, Spectrochim. Acta, Part B 90 (2013) 68-71. doi:10.1016/j.sab.2013.10.008

[23] A.J. Effenberger, J.R. Scott, Practical high-resolution detection method for laser-induced breakdown spectroscopy, Appl. Opt. 51 (7) (2012) B165-B170. doi:10.1364/AO.51.00B165

[24] P. Ko, J.R. Scott, I. Jovanovic, Analysis of high-resolution spectra from a hybrid interferometric/dispersive spectrometer, Opt. Commun. 357 (2015) 95-99.

doi:10.1016/j.optcom.2015.08.077

[25] H.-D.V. Böhm, W. Michaelis, C. Weitkamp, Hyperfine structure and isotope shift measurements on ${ }^{235} \mathrm{U}$ and laser separation of uranium isotopes by two-step photoionization, Opt. Commun. 26 (1978) 177182. doi:10.1016/0030-4018(78)90047-0 


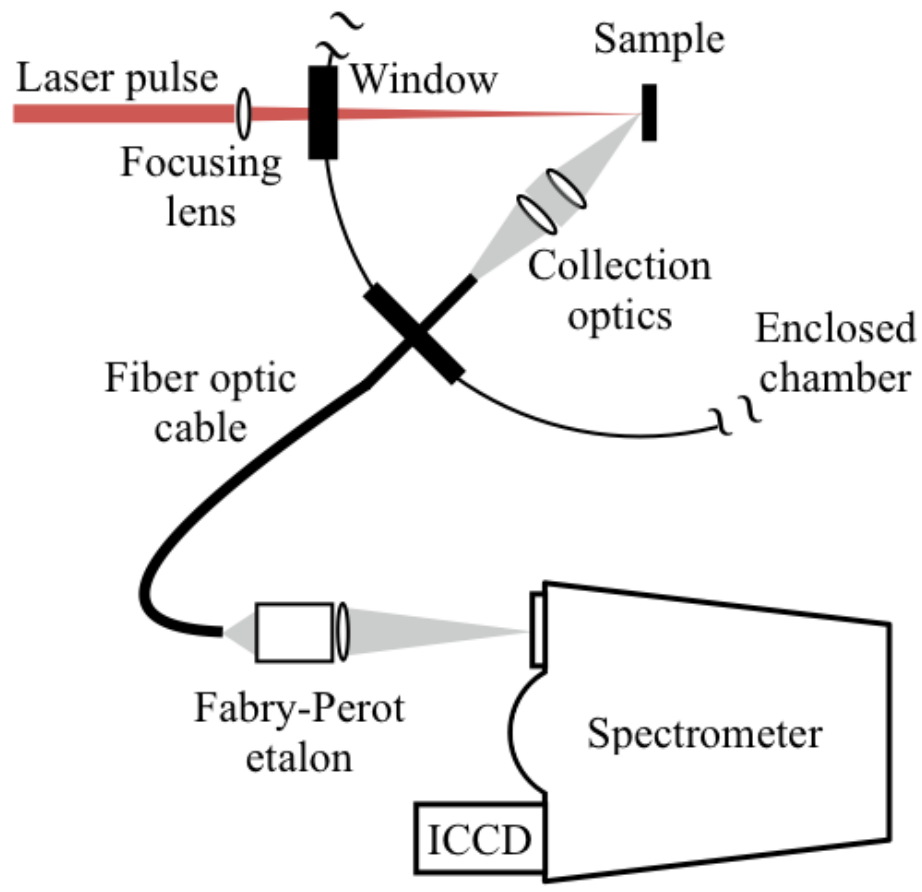

Figure 1: Schematic diagram of experimental setup, laser beam path (red) and transport of light emitted by the plasma (gray). 


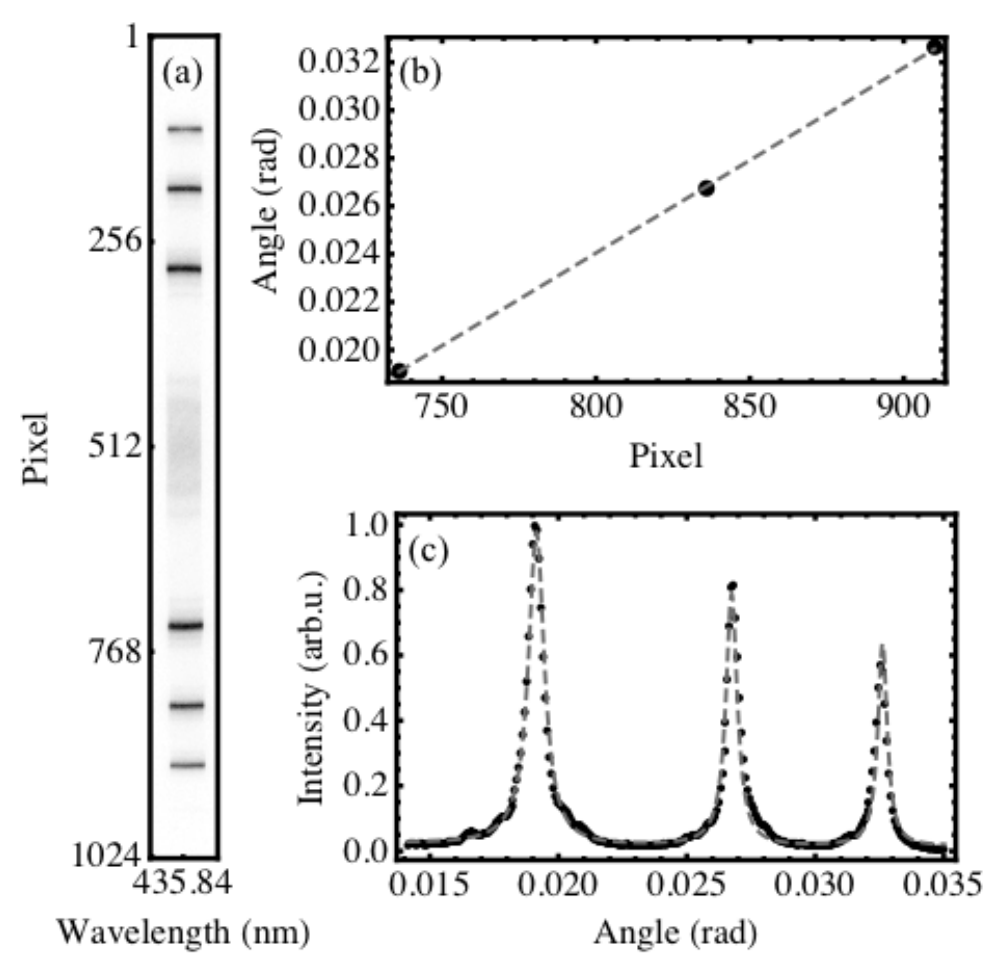

Figure 2: (a) Measured pattern at the wavelength of $435.84 \mathrm{~nm}$; (b) calibration of the angular $(\theta)$ axis using the location of three constructive interference peaks (black markers) and linear fit (dashed line); (c) best fit (dashed line) of the measured interference peaks by Eq. (1) yields the reflectivity of the etalon $(R=0.77)$. Experimentally measured points are shown as black markers. 


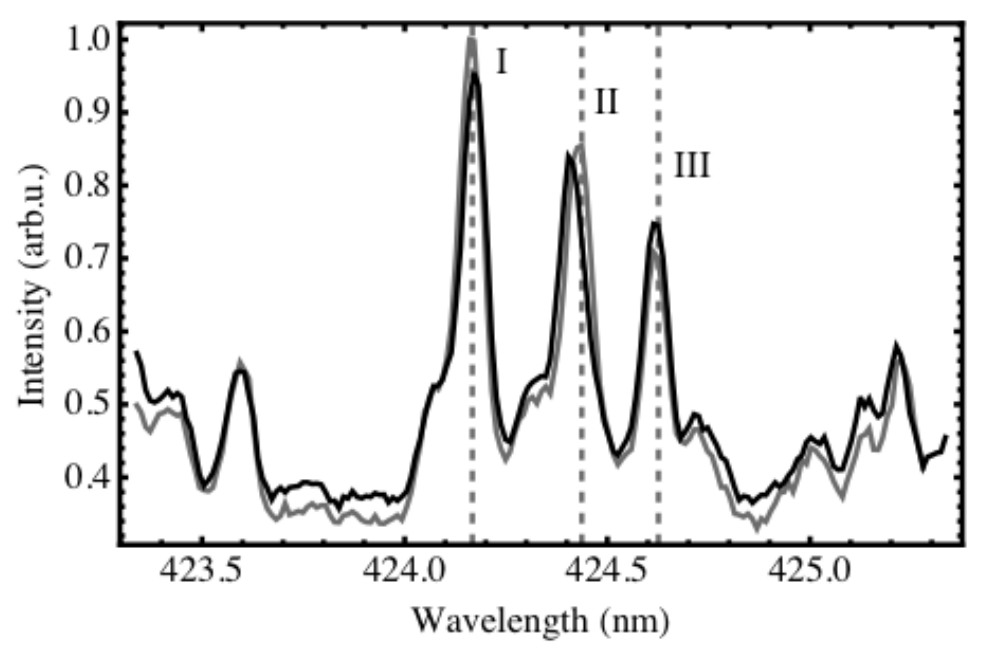

Figure 3: LIBS spectra from natural U (gray line) and HEU (black line) foils measured using a $0.55 \mathrm{~m}$ Czerny-Turner spectrometer alone. The gray dashed lines indicate the positions of ${ }^{238} \mathrm{U}$ lines at $424.167 \mathrm{~nm}$ (I), $424.437 \mathrm{~nm}$ (II), and $424.626 \mathrm{~nm}$ (III). Spectra were normalized for the differences to be more apparent. 

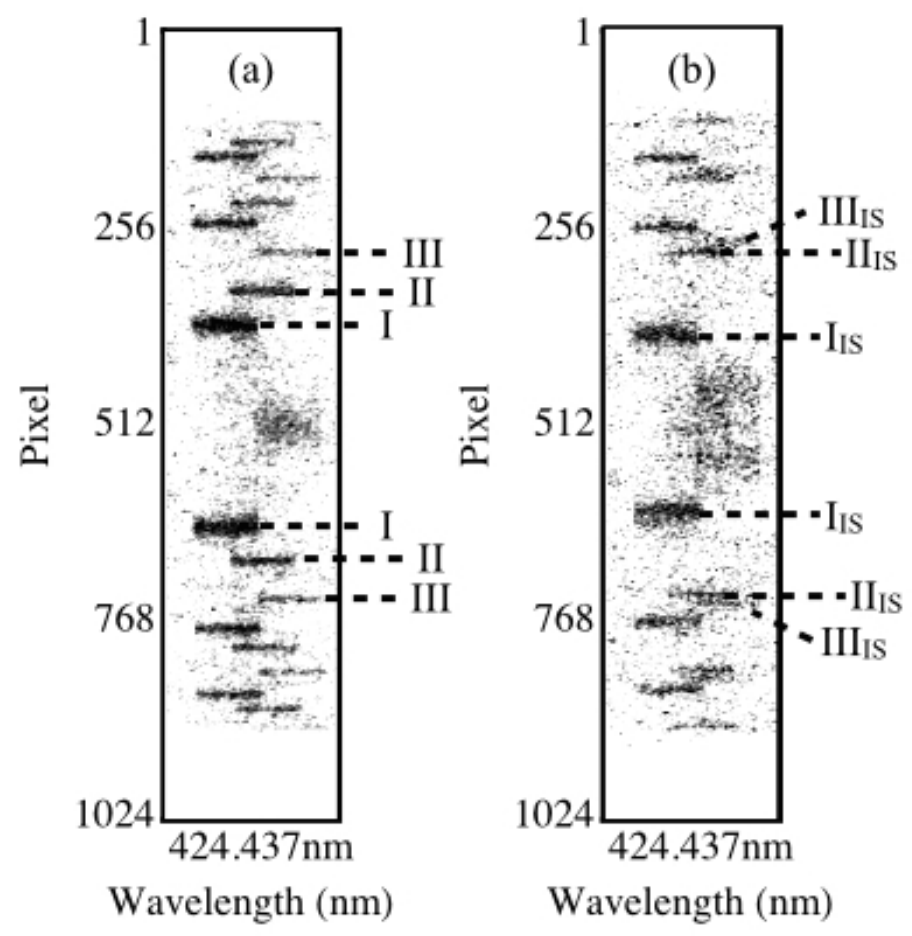

Figure 4: Interference patterns measured using the hybrid FP LIBS system from (a) natural $U$ and (b) HEU sample in the vicinity of $424 \mathrm{~nm}$. The first-order interference pattern of ${ }^{238} \mathrm{U}$ at $424.167 \mathrm{~nm}$ (I), $424.437 \mathrm{~nm}$ (II), and $424.626 \mathrm{~nm}$ (III) lines are indicated in (a); in (b) the isotopically shifted (IS) ${ }^{235} \mathrm{U} 424.173 \mathrm{~nm}\left(\mathrm{I}_{\mathrm{IS}}\right), 424.412 \mathrm{~nm}$ $\left(\mathrm{II}_{\mathrm{IS}}\right)$ and $424.621 \mathrm{~nm}\left(\mathrm{III}_{\mathrm{IS}}\right)$ peaks are marked. Contrast and brightness in the images have been adjusted for publication. 


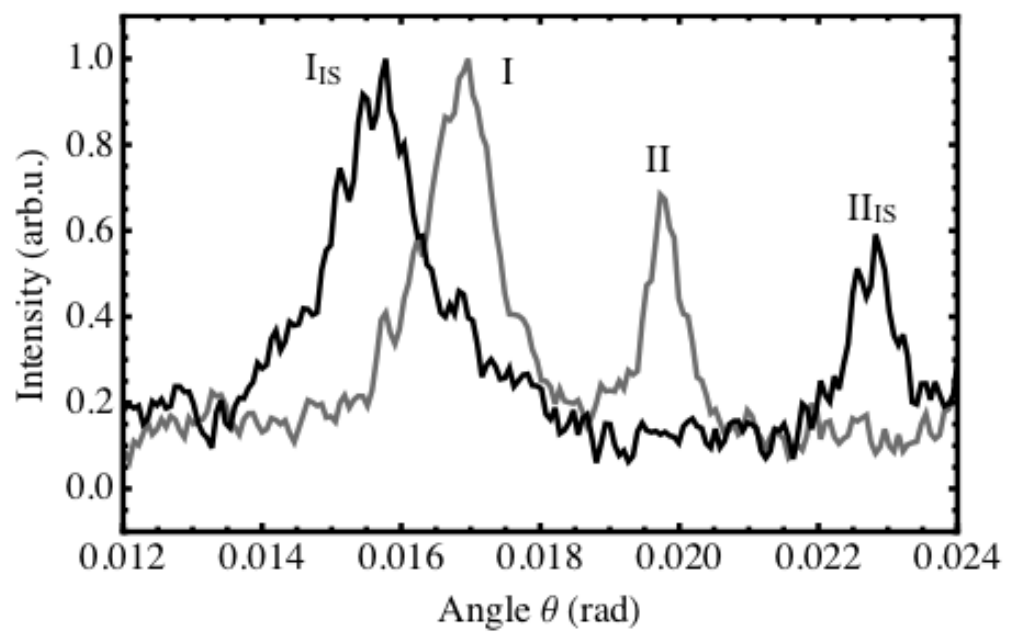

Figure 5: Integrated profile of the natural uranium (gray line) and HEU (black line) interference patterns. The peaks are labeled following the convention set in Fig. 3. Peaks were normalized to show differences between natural and HEU. The $424.626 \mathrm{~nm}$ (III) line and isotope shift are not included in the analysis due to the overlap of the 424.412 $\mathrm{nm}\left(\mathrm{II}_{\mathrm{IS}}\right)$ and $424.676 \mathrm{~nm}\left(\mathrm{III}_{\mathrm{IS}}\right)$ interference lines. 

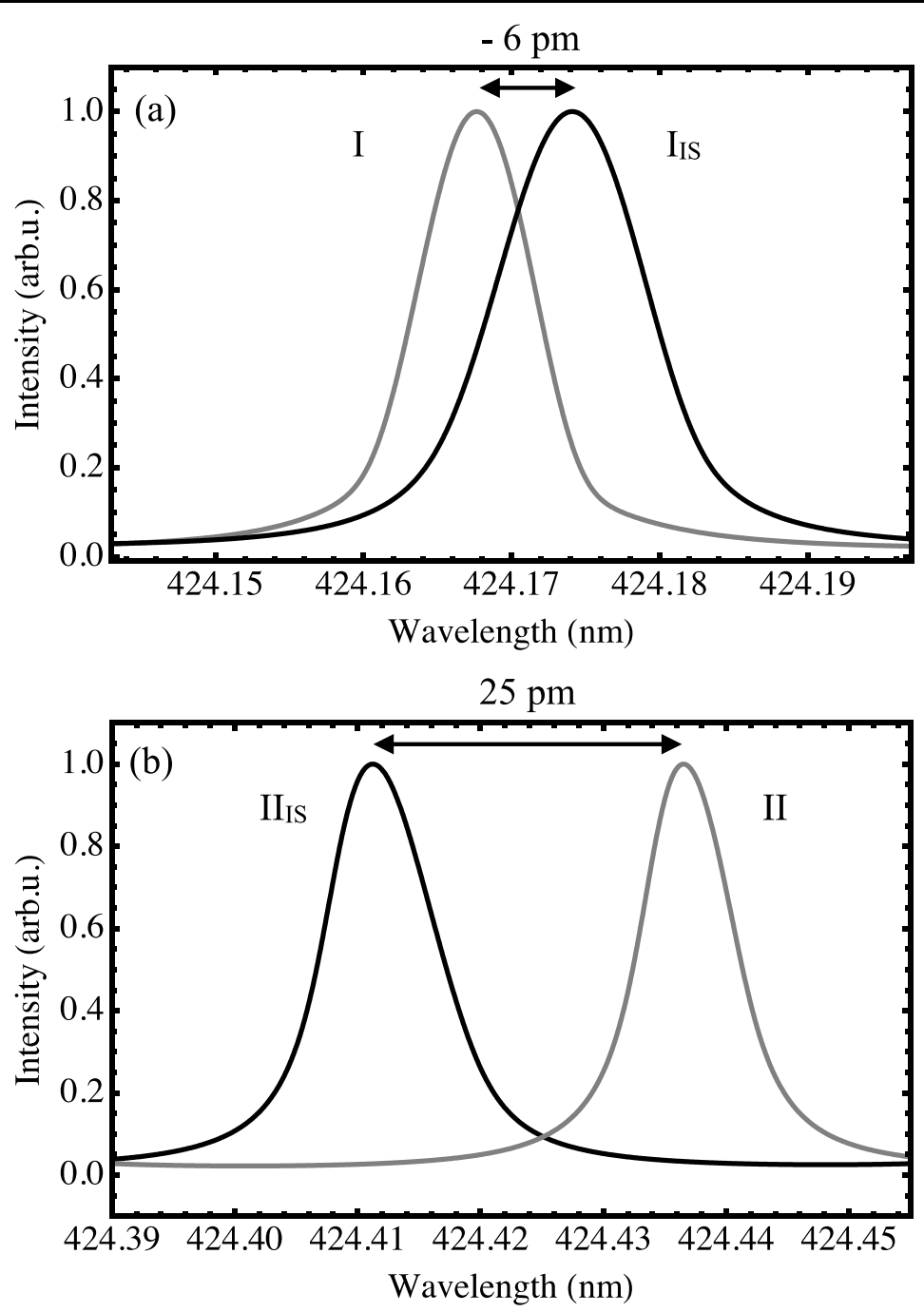

Figure 6: Reconstructed FP LIBS emission spectra of natural uranium (gray line) and HEU (black line) produced using the inversion method. (a) ${ }^{238} \mathrm{U} 424.167 \mathrm{~nm}$ (I) and ${ }^{235} \mathrm{U}$ $424.173 \mathrm{~nm}$ (IIS) lines (-6 pm shift); (b) ${ }^{235} \mathrm{U} 424.412 \mathrm{~nm}$ (IIIS) and ${ }^{238} \mathrm{U} 424.437 \mathrm{~nm}$ (II) lines ( $25 \mathrm{pm}$ shift). Reconstructed spectra were normalized for comparison. 